\title{
Clinical electroencephalogram (EEG) evaluation is improved by the amplitude asymmetry index
}

\author{
Avaliação da assimetria de amplitudes amplia o uso clínico do eletroencefalograma (EEG) \\ Francisco José Carchedi Luccas ${ }^{1}$, Thalita Bártolo², Nayara Lucio da Silva², Barbara Cavenaghi²
}

\begin{abstract}
Cerebral hemispheres, although similar, are neither completely symmetrical in structure nor equivalent in function. EEG asymmetry studies have been directed more to frequency than to amplitude analysis. Objective: Better definition of normal amplitude asymmetry values on the classical EEG frequency bands. Results: EEG amplitude asymmetry index (AAI) is physiologically low in normal adults, differences usually lesser than 7\%. Conclusion: Persistent or intermittent amplitude asymmetry regional differences higher than $7 \%$ may be suggestive of pathology after adequate correlation with clinical data and EEG classical visual analysis.
\end{abstract}

Keywords: Electroencephalography; amplitude; asymmetry.

RESUMO

Os hemisférios cerebrais não são simétricos em morfologia ou funções. Tradicionalmente, os estudos de assimetria do EEG focaram mais na análise de frequências do que na de amplitudes. Objetivo: Contribuir para melhor definição dos valores normais de assimetria de amplitudes nas faixas de frequência clássicas do EEG. Resultados: 0 índice de assimetria de amplitudes é fisiologicamente baixo em adultos, usualmente menor do que 7\%. Conclusão: Se adequadamente correlacionadas aos dados clínicos e à análise visual do EEG, assimetrias persistentes ou intermitentes de amplitude acima de 7\% podem ser sugestivas de patologia.

Palavras-chave: Eletroencfalografia; amplitude; assimetria.

EEG clinical interpretation is based on the detection of paroxysms and on background activity evaluation. Visual analysis is fundamental for paroxysmal detection, not surpassed by computer specific programs. Electrocerebral background activities comprise the dominant rhythms over the scalp, their topography, reactivity, morphology, frequency, amplitude, and only the last two are more amenable to parameterization. Frequency is evaluated by spectral analysis and presents four classical defined bands: delta $(0.5-3.5 \mathrm{~Hz})$, theta $(4-7.5 \mathrm{~Hz})$, alpha $(8-12.5 \mathrm{~Hz})$, and beta $(13-20 \mathrm{~Hz})$. The majority of studies on frequency asymmetry focus on alpha rhythm, considered as pathological whenever values larger than $0.5-1 \mathrm{~Hz}$ are attained. Classical amplitude measures are more laborious, usually done by hand with a ruler, generating subjective criteria of low, medium, and high values ${ }^{1,2,3}$.

The concept of symmetry is ubiquitous in nature and an important property in the evaluation of various clinical conditions.
Cerebral hemispheres, although appearing similar, are neither completely symmetrical in structure nor equivalent in function. Modest physiological asymmetries of EEG rhythms are frequent, but greater asymmetries present clinical relevance in several brain disorders. When based on analogical EEG equipment amplitude asymmetry definition was more difficult: 1) Kugler ${ }^{4}$ referred alpha amplitudes usually higher on the right hemisphere of right-handed individuals, and differences up to $30 \%$ should be considered normal; 2) Kellaway ${ }^{5}$ reported $60 \%$ of adults presenting alpha rhythm voltage asymmetry, half of them with higher values on the right side, not correlated to hemispherical dominance; amplitude differences higher than $50 \%$ should be considered potentially pathologic; 3) Delamonica ${ }^{6}$ reported EEG amplitude as characteristically symmetrical, suggesting to search other parameters, such as frequency, to overcome the dilemma of asymmetry. In general these studies related almost exclusively to alpha rhythm, breach rhythm representing the exception after

${ }^{1}$ Hospital São Luiz, Neurofisiologia Clínica, Neurofisiologia Clínica, São Paulo SP, Brasil;

${ }^{2}$ Hospital São Luiz, Neurofisiologia Clínica, Biomedicina, São Paulo SP, Brasil.

Correspondence: Francisco José Carchedi Luccas; Rua Simão Alvares, 250 / apto. 22; 05417-020 São Paulo SP, Brasil; E-mail: fjluccas@gmail.com

Conflict of interest: There is no conflict of interest to declare.

Received 26 April 2015; Received in final form 18 December 2015; Accepted 18 May 2016. 
surgical burr holes providing beta asymmetry. More recently Aurlien et al. ${ }^{7}$ used visual analysis on occipital leads to measure alpha rhythm separated from the other background activities; the amplitudes showed wide variation, but amplitude asymmetries were not considered.

Brain symmetry index (BSI) developed by Putten ${ }^{2}$ on digital EEG compares longitudinal montage of electrodes on the right and left hemispheres to detect temporal spectral characteristics and interhemispheric asymmetry, but smaller brain regions were not contemplated. BSI was successfully applied in carotid endarterectomy monitoring, ranging from zero (perfect symmetry) to 1 (perfect asymmetry). BSI has also found application monitoring acute strokes and focal seizures in the Intensive Care Unit. Finnigan and van Putten suggested BSI could inform the efficacy of thrombolytic therapy and help prognostication and rehabilitation strategies ${ }^{8,9,10}$.

Numbers (digits) in digital EEG equipment permits the development of several quantitative EEG (qEEG) formats derived by mathematical analysis. But, "Despite the presence of numbers, EEG analyses continue to be limited to classic visual as in the past, taking no advantages of quantitative EEG". Restating, clinical neurophysiologists are recording dEEG, but interpreting solely classical visual xy graphs.
Considering clinical application, should the more recent qEEG be preferred over dEEG graphics? The almost infinite possibility of qEEG formats turns this into a very complex issue. We believe that the simultaneous display of dEEG plus a limited number of qEEG formats represents the best of worlds. Recently a program called Video Monitor EEG EQSA260 (Neurotec Pesquisa \& Desenvolvimento em Biomedicina ${ }^{\circledR}$ ) is being routinely employed, where classical EEG is simultaneously viewed with three digitalized formats: 1) Compressed Spectral Array (CSA), graphics deploying EEG frequency bands spectral power $\left(\mu \mathrm{V}^{2}\right)$ as "hills and valleys" of higher and lower activity. 2) Spectral Edge Frequency (SEF), a cursor defining $90 \%$ of EEG spectral limits. 3) Amplitude asymmetry index (AAI), showing percentage differences among eight electrode pairs of both hemispheres. Better EEG parameters definition may help surpass subjectivity weak limits ${ }^{11}$.

During routine EEG recording an online graphic, seen as a green line, climbs between CSA left and right hemispheres spectral analysis, easily allowing AAI evaluation of every epoch or period ("Raia”) (Figure 1). Artifacts may cause shortlived amplitude variations that are usually disregarded, but when real-time online left or right higher persistent or intermittent drifts are envisaged on a hemisphere or region the quest for pathology is mandatory (Figures 2 and 3 ).

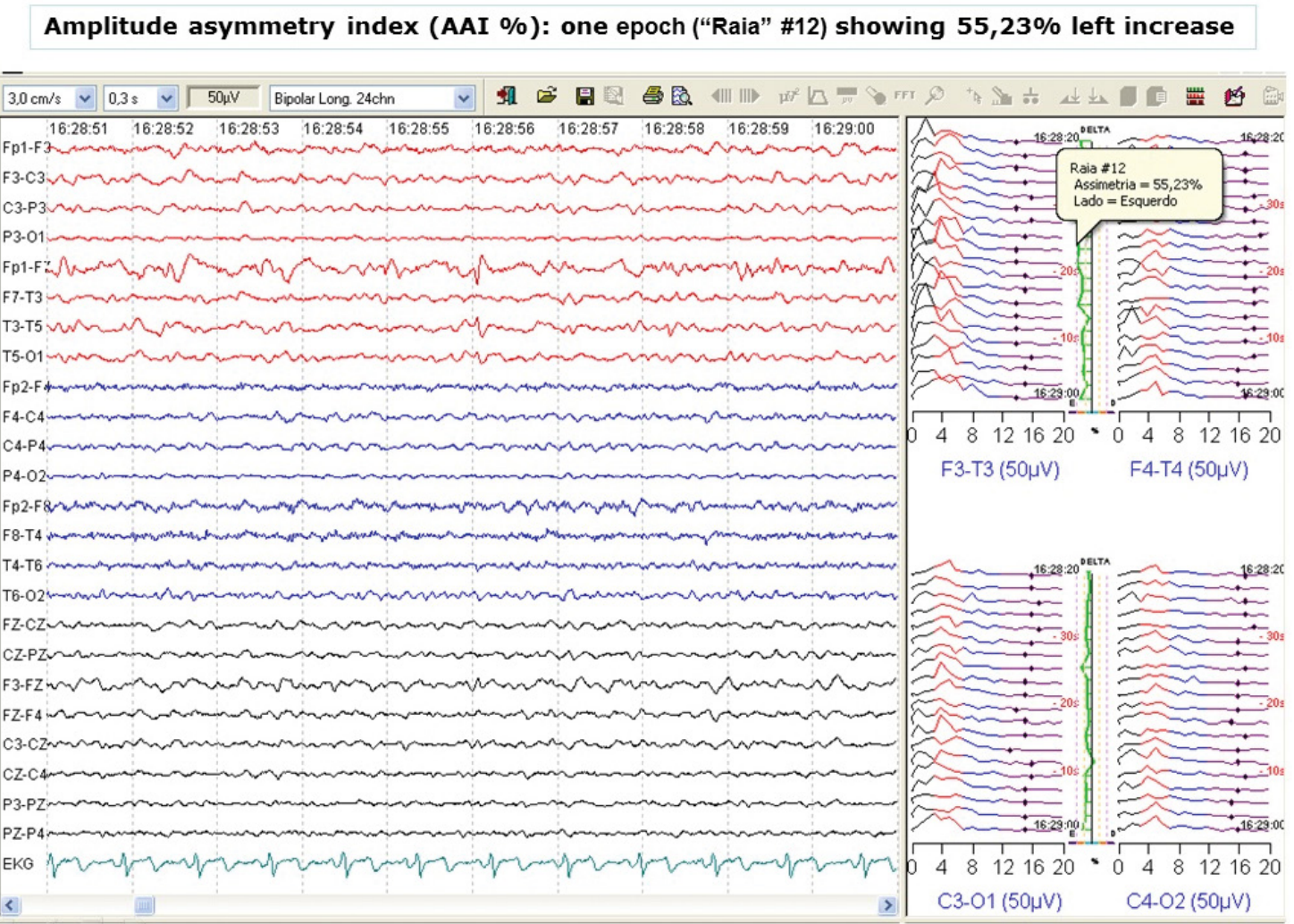

Figure 1. Clinical example showing simultaneous classical electroencephalogram (EEG) channels on the left and Compressed Spectral Array (CSA) analysis on the right. A cursor resting on one of CSA epochs reveal an isolated period amplitude asymmetry percentage (55.23\% "Raia" of 2 seconds). It may be noted that Amplitude Asymmetry Index (AAI) is higher on left anterior (F3-T3 x F4-T4) compared to posterior (C3-01 x C4-02) leads.

i Wilfong AA. Personal communication In 5th Latin-American Clinical Neurophysiology Congress; 2008; Puebla, México. 
Postoperative meningioma: delta increased AAI on the left and more anterior region

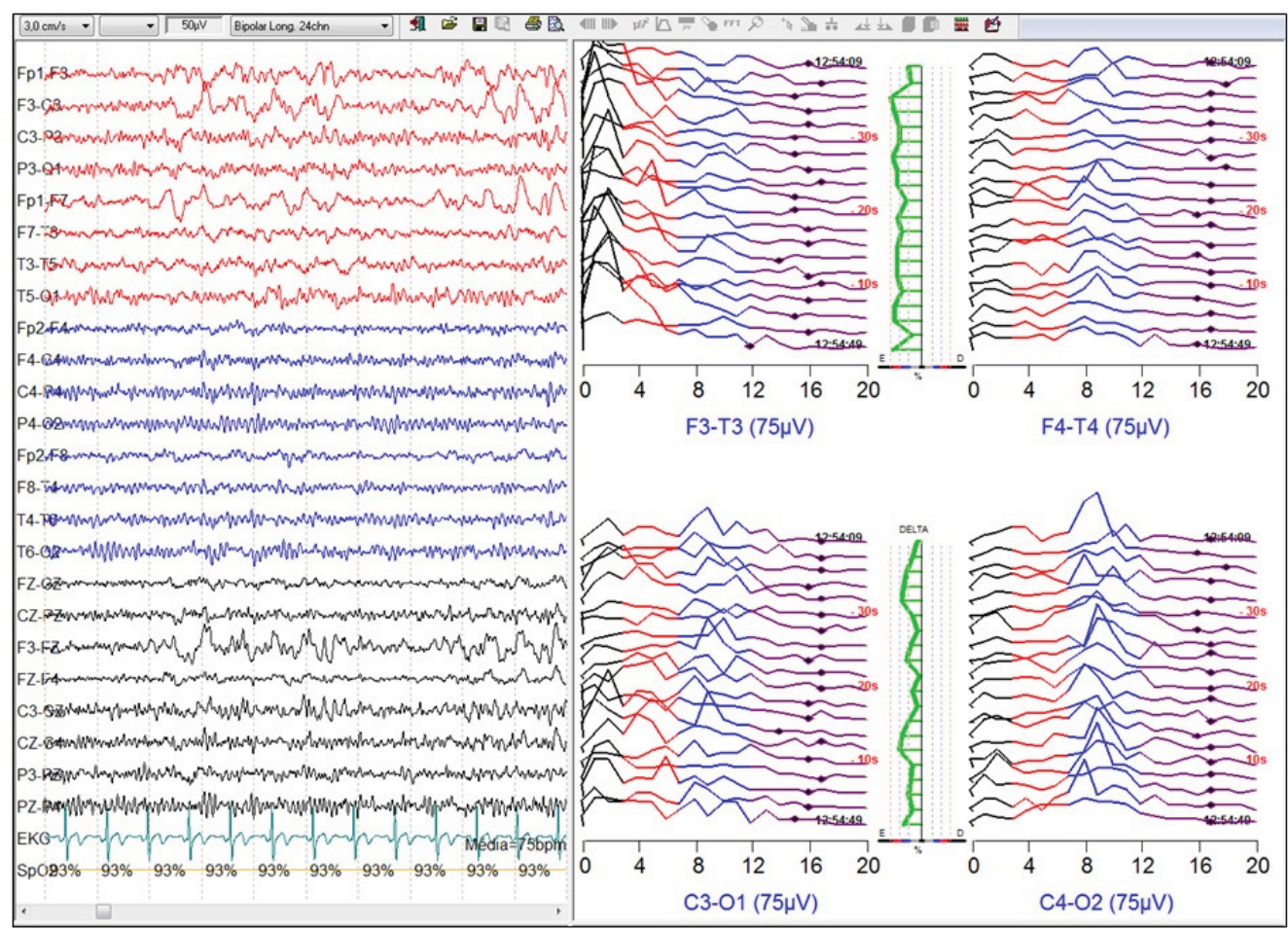

Figure 2. Late postoperative left side meningioma showing: (A) Figure left side, upper 8 channels raw electroencephalogram (EEG) depicting slow delta waves on the left hemisphere, and lower 8 channels with normal EEG on the right hemisphere; (B) Figure right side, Compressed Spectral Array (CSA) comparing F3-T3 to F4-T4 and C3-01 to C4-02; larger amount of delta $\&$ theta slow waves on left hemisphere derivations; (C) Real-time green line between both CSA columns shows a left drift indicating large delta band asymmetry.

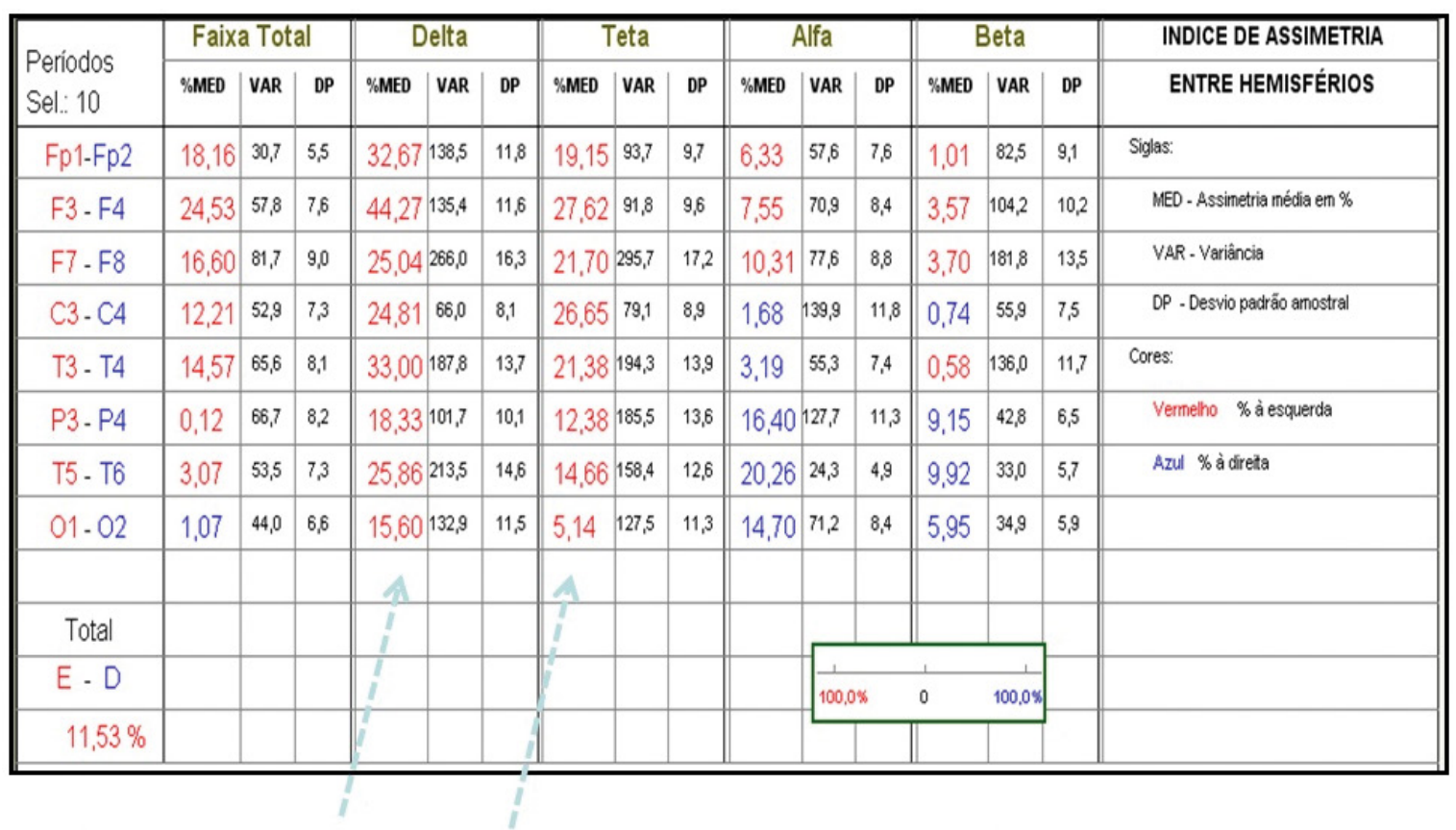

Same patient, offline table showing left delta \& theta increase. Colors: red for left, blue for right $\%$ differences.

Figure 3. Amplitude Asymmetry Index (AAI) table developed offline constituted by 20 artifact-free epochs of the same patient, comparing eight electrode pairs and demonstrating a left side increase of delta \& theta slow waves, compatible with figure 2 online recording. 
An important question will surface whenever lower online AAI values are observed, basically, which are the normal adult acceptable limits? These data were not found in the literature and offline tables expressing the percentage of left-right AAI may help the clinical-EEG correlation. Normal adult AAI values together with few examples will be provided.

\section{METHOD}

Thirty adult patients aged 19 to 59 years (17 F, 13 M) examined at Hospital São Luiz - Morumbi (Figure 4) submitted to EEG as part of their clinical workup had their EEG reported as normal by a team with one clinical neurophysiologist (FJCL) and three biomedical; neurological lesions were not present (Figure 5). Patients with external factors capable of influence symmetry, such as localized scalp edemas or hematomas were excluded. Since EEG recording is noninvasive a written informed consent is considered unnecessary, substituted by a careful explanation of the proceedings, respecting patient questions and agreement.
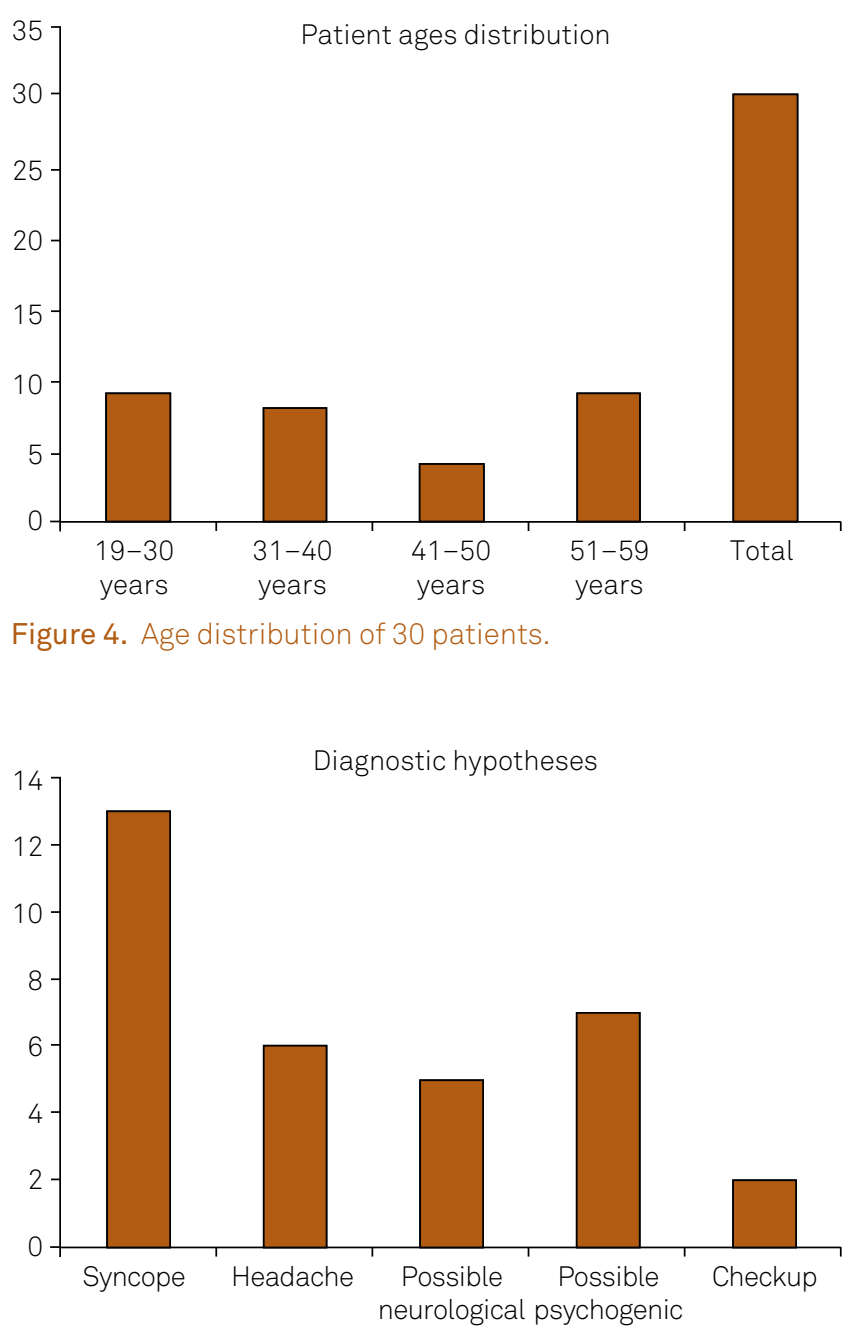

Figure 5. Number of diagnostic hypotheses of 30 patients.
Normal EEG files were retrieved to extract the data, the patients being allocated to three different groups in accordance to temporal recording sequence, ten patients each, whose mean ages were 37.1, 37.4, and 41.5 years.

Noninvasive video-EEG recorded simultaneously 26 channels dEEG plus qEEG CSA, SEF and AAI formats applying 19 scalp and 2 reference linked ears electrodes [A1 + A2] / 2 (10-20 International System) with adhesiveconductive paste, impedance below $5 \mathrm{kOhms}$, plus 2 electrodes for cardiac monitoring, and an oxymeter. Notations were kept for routine eyes open and closed, hyperventilation, and intermittent photic stimulation. Routinely the online AAI is continuously evaluated together with CSA, allowing real-time analysis of a selected frequency band increment or reduction occurring in one hemisphere or region.

After recording offline AAI table allows to compare spectra power percent difference (left minus right) for each frequency band among homologous eight electrode pairs Fp1-Fp2, F3-F4, F7-F8, C3-C4, T3-T4, P3-P4, T5-T6 and O1-O2, also showing sample variation (VAR) and standard deviation (SD), with statistical data provided by the program. Sample variation (VAR) will not be further analysed, it was expectedly large within groups composed of different EEG. AAI SD will also not be further discussed, since lower values neither sustain clinical relevance nor it is accepted that amplitude values sustain Gaussian distribution. Maximal values and their limits represent the searched information.

Development of offline AAI table encompass the following steps: 1) edition of 20 epochs, two seconds each (totaling 40 seconds), selected during wakefulness trying to avoid artifacts; care is taken with artifact exclusion, and comparison with raw EEG may help to overcome identification difficulties with digital formats; 2) activation methods and eyes opening and closing are not included; 3) Fast Fourier Transform (FFT) execution of the 20 selected epochs; 4) the program calculates amplitude percentage difference for every electrode pair on each 2 second epoch, and mean differences along the total 40 seconds time will appear on the asymmetry summary; 5) program sequence is: select "Mapas" (brain maps), “Tabelas" (tables), and "Resumo de assimetria" (asymmetry summary). Each group of ten normal adult EEG generated 200 epochs 20 epochs per EEG and 10 EEG (Figures 6, 7 and 8).

Color brain mapping of absolute frequency bands of the three groups was also analysed, helping to assert the 200 epoch adequate technical quality selection, without gross asymmetries.

An interesting clinical example shows the EEG and AAI table in a patient with breach rhythm ${ }^{12}$.

\section{RESULTS}

Each group developed offline tables (Figures 6, 7 and 8) showing thefour differentfrequencybandsand themeasured 


\begin{tabular}{|c|c|c|c|c|c|c|c|c|c|c|c|c|c|c|c|c|}
\hline \multirow{2}{*}{$\begin{array}{l}\text { Periodos } \\
\text { Sel. } 200\end{array}$} & \multicolumn{3}{|c|}{ Faixa Total } & \multicolumn{3}{|c|}{ Delta } & \multicolumn{3}{|c|}{ Teta } & \multicolumn{3}{|c|}{ Alfa } & \multicolumn{3}{|c|}{ Beta } & \multirow{2}{*}{$\begin{array}{l}\text { INDICE DE ASSIMETRIA } \\
\text { ENTRE HEMISFÉRIOS }\end{array}$} \\
\hline & "SMED & VAR & DP & YMED & VAR & DP & צMED & VAR & DP & หMED & VAR & DP & YMED & VAR & DP & \\
\hline $\mathrm{Fp1} \cdot \mathrm{Fp2}$ & 0,20 & 42,9 & 6,6 & 0,06 & 147,6 & 122 & 1,08 & 92,2 & 9,6 & 0,05 & 56,9 & 7,5 & 0,18 & 142,1 & 11,9 & Sigles: \\
\hline$F_{3} \cdot F_{4}$ & 0,73 & 23,9 & 4,9 & 1,10 & 79,2 & 8,9 & 0,70 & 76,8 & 8,8 & 0,07 & 56,9 & 7,5 & 1,69 & 85,4 & 9,2 & WED - Assinetria média em\% \\
\hline$F 7 \cdot F 8$ & 1,13 & 62,3 & 7,9 & 0,56 & 260,7 & 16,1 & 2,77 & 205,6 & 14,3 & 1,14 & 157,3 & 125 & 0,50 & 104,4 & 102 & VAR-Varininces \\
\hline $\mathrm{C} 3 \cdot \mathrm{C4}$ & 1,34 & 23,9 & 4,9 & 0,96 & 68,0 & 8,2 & 1,37 & 85,0 & 9,2 & 1,04 & 80,6 & 9,0 & 1,35 & 56,5 & 7,5 & DP - Dessio padrio amostral \\
\hline T3 $\cdot$ T4 & 1,51 & 56,6 & 7,5 & 1,27 & 249,9 & 15,8 & 0,89 & 196,6 & 14,0 & 0,92 & 168,6 & 13,0 & 2,04 & 100,1 & 10,0 & Cores: \\
\hline P3 - P4 & 0,82 & 34,0 & 5,8 & 0,15 & 62,6 & 7,9 & 1,32 & 80,1 & 8,9 & 0,57 & 108,0 & 10,4 & 1,07 & 53,8 & 7,3 & Verrmeho \% i esquerda \\
\hline T5 - T6 & 2,33 & 80,5 & 9,0 & 3,86 & 203,2 & 14,3 & 2,71 & 191,3 & 13,8 & 1,83 & 216,6 & 14,7 & 1,03 & 69,2 & 8,3 & Azul \% o drets \\
\hline 01.02 & 0,63 & 40,6 & 6,4 & 2,61 & 95,9 & 9,8 & 1,06 & 77,7 & 8,8 & 1,49 & 80,8 & 9,0 & 1,43 & 42,1 & 6,5 & \\
\hline \multicolumn{17}{|l|}{ Total } \\
\hline$E \cdot D$ & & & & & & & & & & & \multicolumn{2}{|c|}{ 100.0\% } & 0 & $100,0 \%$ & & \\
\hline $0,52 \%$ & & & & & & & & & & & & & & & & \\
\hline $\begin{array}{l}\text { No } \\
\text { Tet } \\
\text { Sig } \\
\text { Ver }\end{array}$ & ( & $\begin{array}{l}\mathrm{fa}= \\
\text { iati }\end{array}$ & ion & 6 ; BeD & $\begin{array}{l}\mathrm{ta}=\mathrm{b} \\
=\mathrm{me}\end{array}$ & n & $\begin{array}{l}s ; E=L \\
\text { dice d } \\
\text { asymm }\end{array}$ & $\begin{array}{l}\text { de A } \\
\text { netr }\end{array}$ & & vari & $\begin{array}{l}\text { aixa To } \\
\text { ntre He } \\
\text { lance; }\end{array}$ & & D, st & $\begin{array}{l}\text { Frec } \\
=\text { Ar } \\
\text { and }\end{array}$ & plit & $\begin{array}{l}\text { Range; Delta = delta; } \\
\text { Asymmetry Index; } \\
\text { ation. Cores = Colors; } \\
\text { ased. }\end{array}$ \\
\hline
\end{tabular}

Figure 6. Amplitude asymmetry index patient group 1.

\begin{tabular}{|c|c|c|c|c|c|c|c|c|c|c|c|c|c|c|c|c|}
\hline \multirow{2}{*}{$\begin{array}{l}\text { Periodos } \\
\text { Sel: } 200\end{array}$} & \multicolumn{3}{|c|}{ Faixa Total } & \multicolumn{3}{|c|}{ Delta } & \multicolumn{3}{|c|}{ Teta } & \multicolumn{3}{|c|}{ Alfa } & \multicolumn{3}{|c|}{ Beta } & \multirow{2}{*}{$\begin{array}{c}\text { INDICE DE ASSIMETRIA } \\
\text { ENTRE HEMISFÉRIOS }\end{array}$} \\
\hline & YMED & VAR & DP & YMED & VAR & $D P$ & YMED & VAR & op & YMED & VAR & $D P$ & SMED & VAR & $D P$ & \\
\hline$F p 1 \cdot F p 2$ & 1,23 & 108,5 & 10,4 & 2,38 & 222,6 & 17,1 & 0,80 & 154,8 & 12,4 & 1,29 & 119,1 & 10,9 & 0,42 & 121,3 & 11,0 & Sigas: \\
\hline$F_{3} \cdot F_{4}$ & 0,52 & 24,3 & 4,9 & 0,48 & 93,1 & 9,6 & 0,10 & 74,3 & 8,6 & 0,36 & 50,1 & 7,1 & 1,57 & 55,0 & 7,4 & UED-Assimetria media em\% \\
\hline F7 . F8 & 2,02 & 51,3 & 7,2 & 1,42 & 272,9 & 16,5 & 2,73 & 205,7 & 14,3 & 0,86 & 126,7 & 11,3 & 2,58 & 83,0 & 9,1 & VAR-Varainces \\
\hline $\mathrm{C} 3 \cdot \mathrm{CA}$ & 2,52 & 16,3 & 4,0 & 1,52 & 83,8 & 9,2 & 2,99 & 70,5 & 8,4 & 3,26 & 63,7 & 8,0 & 2,22 & 32,6 & 5,7 & DP - Desvio padrio amostral \\
\hline $\mathrm{T} 3 \cdot \mathrm{T} 4$ & 4,42 & 66,1 & 8,1 & 2,10 & 218,5 & 14,8 & 3,43 & 246,9 & 15,7 & 5,03 & 179,4 & 13,4 & 4,91 & 14,4 & 11,9 & Cores: \\
\hline$P_{3} \cdot P_{4}$ & 0,61 & 23,3 & 4,8 & 0,48 & 101,6 & 10,1 & 1,41 & 67,0 & 8,2 & 1,14 & 97,6 & 9,9 & 0,89 & 36,5 & 6,0 & Vermeho \%à esquerda \\
\hline T5 - T6 & 2,99 & 98,4 & 9,9 & 3,06 & 191,3 & 13,8 & 1,66 & 214,8 & 14,7 & 4,96 & 209,2 & 16,4 & 0,85 & 98,3 & 9,9 & Azzl \% i dreta \\
\hline 01.02 & 0,87 & 31,0 & 5,6 & 1,50 & 92,3 & 9,6 & 0,05 & 84,4 & 9,2 & 0,06 & 88,8 & 9,4 & 2,04 & 48,4 & 7,0 & \\
\hline \multicolumn{17}{|l|}{ Total } \\
\hline$E \cdot D$ & & & & & & & & & & & \multicolumn{2}{|c|}{$100.0 \%$} & 0 & $\frac{1}{100,0 \%}$ & & \\
\hline $1,39 \%$ & & & & & & & & & & & & & & & & \\
\hline $\begin{array}{l}\text { Notati } \\
\text { Teta = } \\
\text { Siglas } \\
\text { Verme }\end{array}$ & 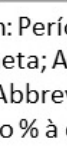 & 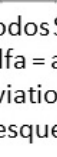 & $\begin{array}{l}\text { Sel } \\
\text { alph } \\
\text { n; } \%\end{array}$ & MED = & $\begin{array}{l}\text { ed e } \\
=b € \\
\text { mea }\end{array}$ & $\%$ & $\begin{array}{l}; E=L \\
\text { dice d } \\
\text { symm }\end{array}$ & etr & $-B i p$ & $\begin{array}{l}\text { hht; Fa } \\
\text { ias En } \\
\text { = varia }\end{array}$ & $\begin{array}{l}\text { ixa To } \\
\text { tre He } \\
\text { ance; }\end{array}$ & $\begin{array}{l}\text { tal }= \\
\text { emisf } \\
\mathrm{P}=\end{array}$ & $\begin{array}{l}\text { Total } \\
\text { férios } \\
\text { SD, st }\end{array}$ & $\begin{array}{l}\text { Frequ } \\
=\text { Amp } \\
\text { andar }\end{array}$ & & $\begin{array}{l}\text { Range; Delta = delta; } \\
\text { Asymmetry Index; } \\
\text { ation. Cores = Colors; } \\
\text { ased. }\end{array}$ \\
\hline
\end{tabular}

Figure 7. Amplitude asymmetry index patient group 2.

AAI percentage (\%MED) for all electrode pairs. Higher left percentages were shown in red and higher right ones in blue. The \%MED comparison shows that AAI percentage is very small in all groups, almost towards symmetry. A color predominance suggesting more left or right AAI values was not observed. The group tables also show variance and standard deviation, but the mixture of 10 different patients prevents its clinical usefulness. 
For a better analyses a new table was compiled depicting only the minimum and maximum percentage AAI (\%MED) values, and minima small values ranged from 0.05-2.73\% with parasagittal (Fp1-Fp2, F3-F4, C3-C4, P3-P4, and O1-O2) electrode pairs predominance (Table - white cells). Intermediate AAI percentages ranging from $2-3 \%$ were sparse (Table - gray cells).

Considering small values close to symmetry carries no pathological decision, more importance will be directed to higher AAI percentage values, which may suggest pathology. Maxima AAI values between $3.73 \%-6.47 \%$ occurred mostly on temporal leads (Table - yellow cells). It is noteworthy to remember that AAI values under $7 \%$ measured in CSA power spectra $\left(\mu \mathrm{V}^{2}\right)$ can be reduced to $2.64 \%$ applying the normal voltage $(\mu \mathrm{V})$ after calculating the root mean square.

Mean minima and maxima values for each frequency band are also shown below on Table; minima mean AAI are below $1 \%$, the lowest mean occurring on alpha band $(0.67 \%)$. The highest mean belongs to delta band (3.16\%).

Careful breach rhythm AAI analysis may present clinical importance after better background activity definition (Figures 9 and 10). Figure 9 show left leads higher EEG amplitude and Figure 10 shows offline table with a left (red) significant increase of physiological rhythms (alpha, beta, and theta) on centrotemporal region and a smaller slow wave delta AAI P3-T3-T5 increase that may be postoperatively tumor related.

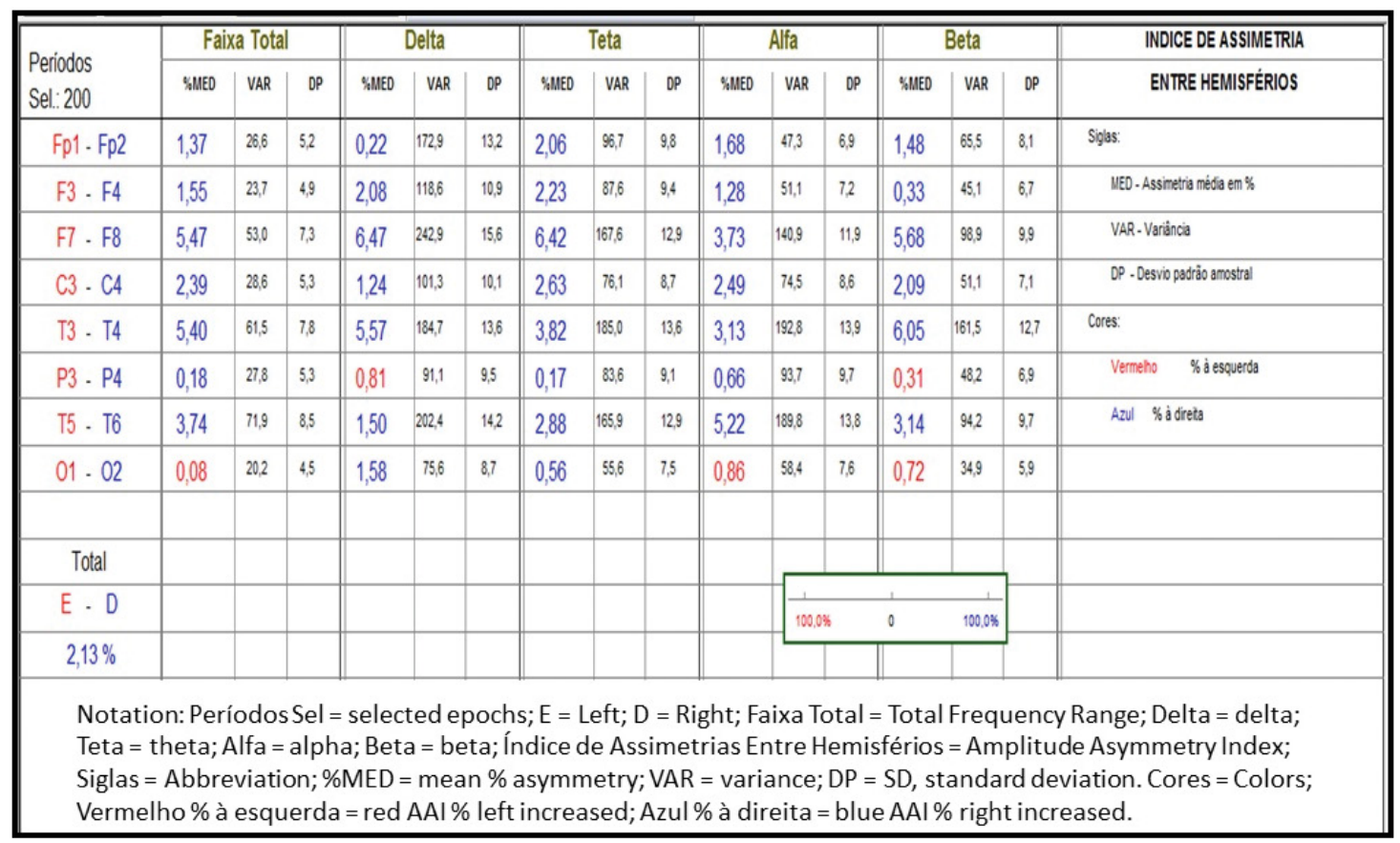

Figure 8. Amplitude asymmetry index patient group 3.

Table. Amplitude asymmetry percentage (AAI) minima and maxima of the different frequency bands.

\begin{tabular}{|c|c|c|c|c|c|c|c|c|c|c|}
\hline \multirow{2}{*}{ Bands } & \multicolumn{2}{|c|}{ Total $0.5-20 \mathrm{~Hz}$} & \multicolumn{2}{|c|}{ Delta $0.5-3.5 \mathrm{~Hz}$} & \multicolumn{2}{|c|}{ Theta $4-7.5 \mathrm{~Hz}$} & \multicolumn{2}{|c|}{ Alpha $8-12.5 \mathrm{~Hz}$} & \multicolumn{2}{|c|}{ Beta $13-20 \mathrm{~Hz}$} \\
\hline & $<$ & $>$ & $<$ & $>$ & $<$ & $>$ & $<$ & $>$ & $<$ & $>$ \\
\hline Fp1-Fp2 & 0.20 & 1.37 & 0.06 & 2.38 & 0.80 & 2.06 & 0.05 & 1.68 & 0.18 & 1.48 \\
\hline F3-F4 & 0.52 & 1.55 & 0.48 & 2.08 & 0.10 & 2.23 & 0.07 & 1.28 & 0.33 & 1.69 \\
\hline C3-C4 & 1.34 & 2.52 & 0.96 & 1.52 & 1.37 & 2.99 & 1.04 & 3.26 & 1.35 & 2.22 \\
\hline P3-P4 & 0.18 & 0.82 & 0.15 & 0.81 & 0.17 & 1.41 & 0.57 & 1.14 & 0.31 & 1.07 \\
\hline 01-02 & 0.08 & 0.87 & 1.50 & 2.61 & 0.05 & 1.06 & 0.06 & 1.49 & 0.72 & 2.04 \\
\hline F7-F8 & 1.13 & 5.47 & 0.56 & 6.47 & 2.73 & 6.42 & 0.86 & 3.73 & 0.50 & 5.68 \\
\hline T3-T4 & 1.51 & 5.40 & 1.27 & 5.57 & 0.89 & 3.82 & 0.92 & 5.03 & 2.04 & 6.05 \\
\hline T5-T6 & 2.33 & 2.99 & 1.50 & 3.86 & 1.66 & 2.88 & 1.83 & 5.22 & 0.85 & 3.14 \\
\hline Mean & 0.91 & 2.62 & 0.81 & 3.16 & 0.97 & 2.85 & 0.67 & 2.85 & 0.78 & 2.92 \\
\hline
\end{tabular}

$<:$ minimum; > maxim 
39 yo $(F)$, postperative left parietal tumor. EEG asymmetry showing breach rhythm on left centrotemporal region (increased amplitude)

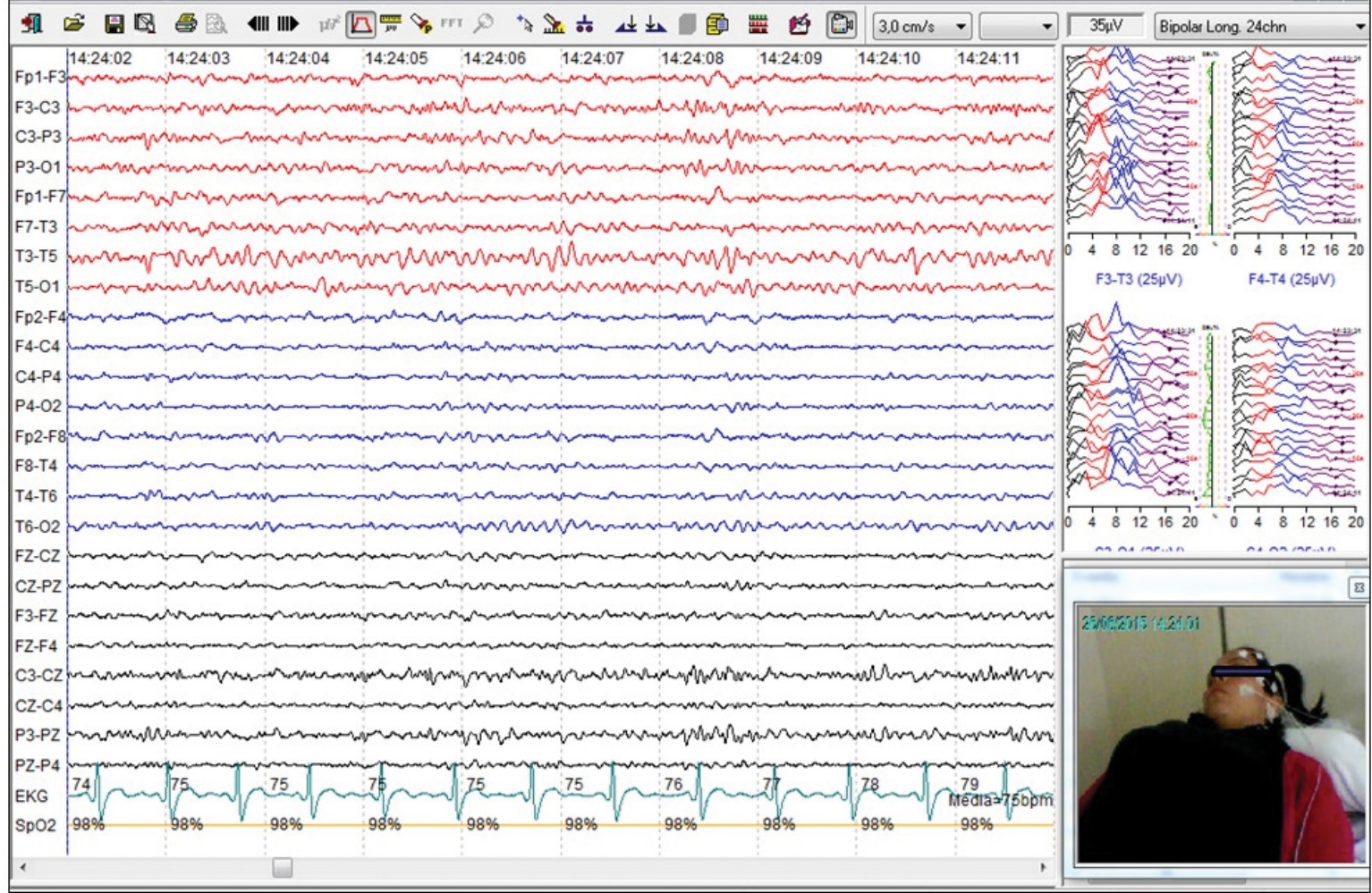

Figure 9. 39 yo (F), postoperative left parietal tumor, showing increased electroencephalogram (EEG) asymmetry amplitude on left centrotemporal region (breach rhythm).

\section{Breach rhythm}

\begin{tabular}{|c|c|c|c|c|c|c|c|c|c|c|c|c|c|c|c|c|}
\hline 螘葍 & 昌夙 & 4 4III & II) & ${ }^{2} \overline{9}$ & $F$ & $\rho$ & 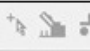 & $\frac{1}{16}$ & \pm & 自 丵 & 幽 & 喅 & $3,0 \mathrm{~cm} / \mathrm{s}$ & $\nabla$ & $0.3 \mathrm{~s}$ & 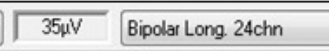 \\
\hline & Faixa & Tot: & & & elta & & & eta & & & $\overline{\text { Alfa }}$ & & & eta & & INDICE DE ASSIMETRIA \\
\hline Sel.: 20 & \%MED & VAR & DP & \%MED & VAR & DP & \%MED & VAR & DP & \%MED & VAR & DP & \%MED & VAR & DP & ENTRE HEMISFÉRIOS \\
\hline Fp1-Fp2 & 1,05 & 55,8 & 7,5 & 0,92 & 411,4 & 20,3 & 1,04 & 69,8 & 8,4 & 1,14 & 54,1 & 7,4 & 2,86 & 42,6 & 6,5 & Siglas: \\
\hline $\mathrm{F} 3-\mathrm{F} 4$ & 5,99 & 19,4 & 4,4 & 1,59 & 127,9 & 11,3 & 4,14 & 52,7 & 7,3 & 6,51 & 50,5 & 7,1 & 11,07 & 61,5 & 7,8 & MED - Assimetria média em \% \\
\hline F7 - F8 & 2,89 & 37,8 & 6,1 & 1,67 & 199,9 & 14,1 & 5,54 & 217,9 & 14,8 & 2,05 & 255,1 & 16,0 & 3,70 & 89,1 & 9,4 & VAR - Variância \\
\hline C3 - C4 & 9,59 & 15,8 & 4,0 & 6,77 & 116,3 & 10,8 & 4,83 & 66,3 & 8,1 & 11,41 & 73,2 & 8,6 & 15,53 & 46,8 & 6,8 & DP - Desvio padrão amostral \\
\hline T3 - T4 & 16,46 & 37,1 & 6,1 & 11,48 & 169,4 & 13,0 & 13,20 & 176,7 & 13,3 & 21,07 & 241,4 & 15,5 & 15,53 & 87,4 & 9,3 & Cores: \\
\hline P3 - P4 & 12,20 & 21,1 & 4,6 & 12,52 & 104,2 & 10,2 & 7,24 & 58,3 & 7,6 & 12,43 & 77,6 & 8,8 & 17,04 & 97,9 & 9,9 & Vermelho \% à esquerda \\
\hline $\mathrm{T} 5-\mathrm{T} 6$ & 18,56 & 23,6 & 4,9 & 17,68 & 185,3 & 13,6 & 14,15 & 128,3 & 11,3 & 22,43 & 118,4 & 10,9 & 17,23 & 99,1 & 10,0 & Azul \% à direta \\
\hline $01-02$ & 5,90 & 15,4 & 3,9 & 6,28 & 55,0 & 7,4 & 1,20 & 88,5 & 9,4 & 7,12 & 104,1 & 10,2 & 10,79 & 67,2 & 8,2 & \\
\hline Total & & & & & & & & & & & & & & & & \\
\hline$E \cdot D$ & & & & & & & & & & & 100,09 & & 0 & $\frac{1}{100.0 \% 6}$ & & \\
\hline $9,25 \%$ & & & & & & & & & & & & & & & & \\
\hline r & st & 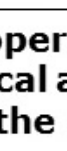 & tar. & off & do & & le & $t$ & 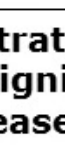 & ng & $\mathbf{D}^{-}$ & & ea & r & vtl & $\begin{array}{l}\text { aspects: } \\
\text { centrotemporal } \\
\text { imor related }\end{array}$ \\
\hline
\end{tabular}

Figure 10. Postoperative breach rhythm offline table illustrating physiological alpha, beta and theta significant Amplitude Asymmetry Index (AAI) increase over left centrotemporal region; there is also a smaller delta AAl increase on P3-T3-T5 that may be tumor related. 


\section{DISCUSSION}

International 10-20 system of EEG electrodes placement based on bone and facial landmarks uses proportional distances as a standard guideline in clinical neurophysiology. The system was predominantly developed for major sulci, but do not exactly represent the subjacent cerebral areas. Myslobodsky et al. ${ }^{12}$ showed enormous differences between electrode position and markers such as the longitudinal fissure and falx position, the inion localization, and the relationship between temporal electrodes and sylvian fissure. Indications also exist that EEG asymmetries are related to skull thickness and to the amount of cortex buried in the fissures of the two sides ${ }^{12}$.

EEG frequency bands asymmetries historically received more attention than amplitudes, considered far less important, and on occasion it was assumed that hemispherical (skull) asymmetries do not exert a major role in routine EEG.

Envisaging automated EEG analysis development, BSI was employed together with other parameters such as alpha rhythm frequency, reactivity, anteroposterior gradients, asymmetries, and diffuse slow-wave activity. Nevertheless, the automated system presents some drawbacks when considering amplitude asymmetries: different hemispherical regions were not individualized, amplitude asymmetries on other frequency bands were not discussed, lack of exact definition of zero (perfect symmetry) and 1 (perfect asymmetry), and a rather subjective affirmation of "symmetric", "slightly asymmetric", or "asymmetric" without defining the limits ${ }^{2,8}$.

Initially intended for critical care application, it was soon decided to routinely apply the Video Monitor EEG EQSA260 program to all patients, and our work derived from clinical EEG routine observation of occasionally larger online amplitude asymmetry values. Adequately correlated to localized brain lesions diagnosis, routine EEG analysis simultaneously recorded with CSA spectral analysis, accompanied by online amplitude asymmetry index permit better characterize the EEG. To attain better parameterization and define normal adult AAI limits, an offline table was created to demonstrate differences among electrode pairs on classical EEG frequency bands. Whenever a more precise AAI is necessary a quick table is offline constructed, helping to observe on which frequency bands the asymmetry is present. Correlation of these formats simultaneously recorded with dEEG well known established values may amplify clinical neurophysiology information. AAI is physiologically low in normal adults, some regions (parasagittal) with values below $2 \%$, and not surpassing $7 \%$ even on regions where it is higher (temporal). AAI values exceeding $7 \%$ may be helpful to detect the occurrence of lower grade asymmetries.

EEG after craniotomy is difficult to interpret because of the presence of a breach rhythm consisting of unfiltered higher voltage physiological waveforms, sometimes with a spiky morphology and frequently associated with a few slow waves mimicking abnormalities. Brigo et al. ${ }^{13}$ recommended the adoption of a "conservative" reading, not misinterpreting the EEG as pathological. AAI offline tables permit more detailed definition of the breach rhythm composition, helping to reduce errors ${ }^{13}$.

Future studies may be directed to compare two successive EEG of the same patient, refining the concept of improvement or deterioration.

\section{Acknowledgements}

Dr. Luiz Calistro Balestrassi supplied the EEG program. Dr. Daniel Cibils made valuable suggestions concerning the study of EEG symmetry.

\section{References}

1. Niedermeyer E. The normal EEG of the waking adult. In: Niedermeyer E, Silva FL, editors. Electroencephalography: basic principles, clinical applications and related fields. 4th ed. Philadelphia: Lippincott Williams \& Wilkins; 1999. p. 149-73.

2. Putten MJAM. The revised brain symmetry index. Clin Neurophysiol. 2007;118:2362-7.

3. Kandel ER. Brain and behavior. In: Kandel ER, Schwartz JH, Jessell TM. Principles of neural science, editors. 3rd Ed. New York: Elsevier; 1991. p. 1-17.

4. Kugler J. La electroencefalografia em la clínica y em la practica. Madrid: Alhambra; 1969. El electroebcefakigrana: elementos gráficos: nomenclatura; p. 39-57.

5. Kellaway P. An orderly approach to visual analysis: the parameters of the normal EEG in adults and children. In: Klass DW, Daly DD, editors., Current practice of clinical electroencephalography. New York: Raven Press; 1979. p. 69-147.

6. Delamonica EA. Electroencefalografía. 2nd ed. Buenos Aires: Libreria El Ateneo; 1984. Chapter 4, El EEG Normal del Adulto; p. 87-130.

7. Aurlien H, Gjerde IO, Aaserth JJ, Eldoen G, Karlsen, B, Skeidsvoll H et al. EEG background activity described by a large computerized database. Clin Neurophysiol. 2004;115(3):665-73. doi:10.1016/j.clinph.2003.10.019
8.

Cloostermans MC, Vos CC, Putten MJAM. A novel approach for computer assisted EEG monitoring in the ICU. Clin Neurophysiol. 2011;122(10):2100-9. doi:10.1016/j.clinph.2011.02.035

9. Finnigan S, Putten MJAM. EEG in ischaemic stroke: quantitative EEG can uniquely inform (sub)-acute prognoses and clinical management. Clin Neurophysiol. 2013;124(1):10-19. doi:10.1016/j.clinph.2012.07.003

10. Lodder SS, Putten MJAM. Quantification of the adult EEG background pattern. Clin Neurophysiol. 2013;124(2):228-37. doi:10.1016/j.clinph.2012.07.007

11. Bickford RG, Billinger TW, Fleming NI, Stewart F. The compressed spectral array (CSA): a pictorial EEG. Proc San Diego Biomed Symp. 1972;11:365-70.

12. Myslobodsky MS, Coppola R, Bar-Ziv J, Weinberger DR. Adequacy of the International 10-20 Electrode System for Computed Neurophysiologic Topography. J Clin Neurophysiol. 1990;7(4):507-18.

13. Brigo F, Cicero R, Fiaschi A, Bongiovanni LG. The breach rhythm. Clin Neurophysiol. 2011;122(11):2116-20. doi:10.1016/j.clinph.2011.07.024 